\title{
Early neonatal sepsis with the extended spectrum $\beta$-lactomase producing: Morganella morgagni
}

\author{
Arvind Sehgal* and David Metz \\ Monash Newborn, Monash Medical Centre, Clayton, VIC, Australia
}

\section{Introduction}

Early onset neonatal sepsis is known to contribute to neonatal mortality and morbidity. Morganella morgagni, an extended spectrum $\beta$-lactamase (ESBL) producing organism, is an uncommon gram negative bacterium in neonates and can cause severe systemic infection. This report presents one such case in which maternal symptoms started 48 hours after delivery. Issues of potential emergence of antibiotic resistance are highlighted.

\section{Case report}

This male infant was born at a level II institution at 38 weeks' gestation and weighed $2540 \mathrm{~g}$. Pregnancy was uneventful except for unexplained intrauterine growth restriction (IUGR). Labor was induced for IUGR and membranes were ruptured 5 hours prior to delivery with clear amniotic fluid. The mother received penicillin in view of Group B Streptococcus (GBS) growth on vaginal swab. Baby was delivered by emergency caesarean section due to fetal bradycardia. Apgar scores were 4 and 7 at one and five minutes respectively while cord arterial $\mathrm{pH}$ was 7.25 and lactate 3.2. After initial sta-

* Corresponding author: Dr. Arvind Sehgal, Monash Newborn, Monash Medical Centre, 246 Clayton Road, Clayton, VIC 3168 Australia. Tel.: +613 95945197; Fax: +613 95946115; E-mail: Arvind.Sehgal@Southernhealth.org.au. bilization and ventilation, he was transferred to the tertiary unit. Soon after arrival, he developed oxygenation failure (Oxygenation index OI - 20.2) and had echocardiographic features consistent with pulmonary hypertension (bidirectional ductal shunt and tricuspid regurgitant jet) and was administered inhaled nitric oxide (iNO) and received inotropes. A frontal CXR was done which showed right lower lobe consolidation. Acute phase reactants like $\mathrm{C}$ reactive protein (CRP) were elevated ( $24 \mathrm{mg} / \mathrm{L}$, normal range $0-5 \mathrm{mg} / \mathrm{L}$ ) and immature to total neutrophil ratio of 0.42 (normal $<0.2$ ), with toxic granulations and vacuolization on peripheral smear. He was initially started on intravenous penicillin/ gentamicin and cefotaxime was added after admission to the tertiary center, considering deterioration in the clinical condition. Blood culture taken at the referring hospital grew Morganella morgagni, resistant to Amoxicillin and Amoxicillin/Clavulanate and sensitive to Cefotaxime, Gentamicin and Meropenem. Lumbar puncture done on day two was normal. Antibiotics were changed to Imipenem and Gentamicin. Placental histopathological examination showed no evidence of funisitis, villitis or chorioamnionitis. Two days after delivery, mother developed high grade fever $\left(39^{\circ} \mathrm{C}\right)$ with chills and rigors and on investigation, grew Morganella morgagni in urine and was started on oral trimethoprim.

\section{Discussion}

This case report presents a term infant with early onset sepsis with Morganella morgagni. This organ- 
ism usually presents as a nosocomial infection in immunocompromised hosts or those with chronic urinary catheterization [1]. The bacterium has been previously implicated in urinary and respiratory tract infections as well as skin and soft tissue infections in adults. A case of neonatal brain abscess has been previously reported which needed surgical treatment and antibiotics [2]. It is an uncommon isolate in the neonatal population, most cases being reported in preterm infants with evidence of maternal chorioamnionitis [3]. In the present case, there were no indicators of antenatal maternal sepsis, other than GBS colonization. Normal placental histopathology speaks strongly against any chorioamnionitis. However, absence of inflammatory changes of the placenta, membranes and umbilical cord certainly does not preclude contact with or aspiration of heavily colonized fluid from the birth canal at or shortly after the time of membrane rupture.

While antenatal treatment with ampicillin/amoxicillin has been shown to reduce the risk of GBS infection in the infant, there are concerns that it may lead to emergence of resistant Gram negative organisms due to the selection of naturally ampicillin resistant organisms such as Morganella [3-5]. While intrapartum antibiotic prophylaxis recommendations are based on population based studies, and have lead to an appreciable decline in GBS sepsis, there is an urgent need to address the issue of effects of this strategy on the changing spectrum of isolates. Rational rather than liberal use of antibiotics could be a first step. Like Enterobacteriaceae spp., Morganella morgagni has a chromosomally encoded and inducible AmpC (ß)-lactamase, which confers resistance to those beta lactam antibiotics (e.g. Ampicillin, third generation cephalosporins) that induce its synthesis by derepression of AmpC [3]. The resulting "derepressed" mutants are resistant to third generation cephalosporins, leading to potential unsuccessful therapy. Selection of this type is reported in $20-30 \%$ of Enterobacter pneumonia and bacteremia cases. It poses a serious public health issue, since only a limited number of antimicrobial agents can be used effectively against the organisms that produce both ES$\mathrm{BL}$ and $\mathrm{AmpC}(B)$ - lactamase [6]. In view of this real risk of emergence of resistance, therapy with third generation cephalosporins should be reconsidered in presence of Morganellagrowth, especially if the baby continues to be symptomatic. In a study on 493 Gram negative isolates, 50 of which were Morganella, the majority of strains were sensitive to Cefipime (97.8\%) and Imipenem (99.6\%) [6], hence these could be antibiotics of choice when faced with organisms like Morganella.

In summary, this case report highlights the role of Morganella morgagnias an uncommon pathogen in neonatal septicemia with a focus on choice of antibiotic therapy in such cases.

\section{References}

[1] J. Tena, Pyomyositis caused by Morganella morgagniin a patient with AIDS, Clin Infect Dis 22 (1996), 372-373.

[2] M. Verboon-Maciolek, W.P. Vandertop, A.C.B. Peters, J.J. Roord and S.P.M. Geelen, Neonatal brain abscess caused by Morganella morgagni, Clin Infect Dis 20 (1995), 471.

[3] A.K. Sinha, S.T. Kempley, E. Price, B.K. Sharma and D. Livermore, Early onset Morganella morgagni sepsis in a newborn infant with emergence of cephalosporin resistance caused by derepression of AmpC-B- lactamase production, Pediatr Infect Dis J 25 (2006), 376-377.

[4] J.L. Rowen and S.M. Lopez, Morganella morgagnisepsis early onset sepsis, Pediatr Infect Dis J 17 (1998), 1176-1177.

[5] T.A. Joseph, S.P. Pyati and N. Jacobs. Neonatal early onset Escherichia coli disease: the effect of intrapartum ampicillin, Arch Pediatr Adolesc Med 152 (1990), 35-40.

[6] S.H. Choi, J.E. Lee, S.J. Park, M.N. Kim, E.J. Choo, Y.G. Kwak et al., Prevalence, microbiology, and clinical characteristics of extended-spectrum B-lactamase producing Enterobacter spp Serratia marcescens Cirobacter freundii and Morganella morgagni in Korea, Eur J Clin Microbiol Infect Dis 26 (2007), 557-561. 